\title{
FACTORIZATION SEMIGROUPS AND IRREDUCIBLE COMPONENTS OF HURWITZ SPACE. II
}

\author{
VIK.S. KULIKOV
}

\begin{abstract}
This article is a continuation of the article with the same title (see arXiv:1003.2953v1). Let $\operatorname{HUR}_{d, t}^{G}\left(\mathbb{P}^{1}\right)$ be the Hurwitz space of degree $d$ coverings of the projective line $\mathbb{P}^{1}$ with Galois group $G$ and having fixed monodromy type $t$ consisting of a collection of local monodromy types (that is, a collection of conjugacy classes of permutations $\sigma$ of the symmetric group $\mathcal{S}_{d}$ acting on the set $\left.I_{d}=\{1, \ldots, d\}\right)$. We prove that if the type $t$ contains big enough number of local monodromies belonging to the conjugacy class $C$ of an odd permutation $\sigma$ which leaves fixed $f_{C} \geqslant 2$ elements of $I_{d}$, then the Hurwitz space $\mathrm{HUR}_{d, t}^{\mathcal{S}_{d}}\left(\mathbb{P}^{1}\right)$ is irreducible.
\end{abstract}

\section{INTRODUCTION}

This article is a continuation of article [1]. To formulate the results of presented article, let us recall main definitions and notations used in [1]. A collection $(S, G, \alpha, \rho)$, where $S$ is a semigroup, $G$ is a group, and $\alpha: S \rightarrow G, \rho: G \rightarrow \operatorname{Aut}(S)$ are homomorphisms, is called a semigroup $S$ over a group $G$ if for all $s_{1}, s_{2} \in S$ we have

$$
s_{1} \cdot s_{2}=\rho\left(\alpha\left(s_{1}\right)\right)\left(s_{2}\right) \cdot s_{1}=s_{2} \cdot \lambda\left(\alpha\left(s_{2}\right)\right)\left(s_{1}\right),
$$

where $\lambda(g)=\rho\left(g^{-1}\right)$. Let $\left(S_{1}, G, \alpha_{1}, \rho_{1}\right)$ and $\left(S_{2}, G, \alpha_{2}, \rho_{2}\right)$ be two semigroups over a group $G$. A homomorphism of semigroups $\varphi: S_{1} \rightarrow S_{2}$ is said to be defined over $G$ if $\alpha_{1}(s)=\alpha_{2}(\varphi(s))$ and $\rho_{2}(g)(\varphi(s))=\varphi\left(\rho_{1}(g)(s)\right)$ for all $s \in S_{1}$ and $g \in G$.

A pair $(G, O)$, where $O \subset G$ is a subset of a group $G$ invariant under the inner automorphisms, is called an equipped group. To each equipped group $(G, O)$ one can associate a semigroup $S_{O}=S(G, O)$ over $G$ (called the factorization semigroup of the elements of $G$ with factors in $O$ ) generated by the elements of the alphabet $X=X_{O}=\left\{x_{g} \mid g \in O\right\}$ being subject to the following relations:

$$
x_{g_{1}} \cdot x_{g_{2}}=x_{g_{2}} \cdot x_{g_{2}^{-1} g_{1} g_{2}}=x_{g_{1} g_{2} g_{1}^{-1}} \cdot x_{g_{1}}
$$

for each $x_{g_{1}}, x_{g_{2}} \in X$ and if $g_{2}=\mathbf{1}$ then $x_{g_{1}} \cdot x_{\mathbf{1}}=x_{g_{1}}$. The map $\alpha: X \rightarrow G$, given by $\alpha\left(x_{g}\right)=g$ for each $x_{g} \in X$, induces a homomorphism $\alpha: S_{O} \rightarrow G$ called the product homomorphism. The action (from the left) $\rho$ of the group $G$ on $S_{O}$ is defined by the action on the alphabet $X$ as follows:

$$
x_{a} \in X \mapsto \rho(g)\left(x_{a}\right)=x_{g a g^{-1}} \in X
$$

for each $g \in G$. Note that $\alpha(\rho(g)(s))=g \alpha(s) g^{-1}$ for all $s \in S_{O}$ and all $g \in G$.

This research was partially supported by grants of NSh-4713.2010.1 and RFBR 08-01-00095. 
Let $O \backslash\{\mathbf{1}\}=C_{1} \sqcup \cdots \sqcup C_{m}$ be the decomposition of the set $O$ into the disjoint union of conjugacy classes of elements of $G$. Each element $s=x_{g_{1}} \cdot \ldots \cdot x_{g_{n}} \in S_{O}$ defines an element $\tau(s)=n_{1} C_{1}+\cdots+n_{m} C_{m}$ of the free abelian semigroup generated by $C_{1}, \ldots, C_{m}(\tau(s)$ is called the type of $s)$, where $n_{i}$ is equal to the number of factors $x_{g_{j}}$ entering in the factorization $s=x_{g_{1}} \cdot \ldots \cdot x_{g_{n}}$ for which $g_{j} \in C_{i}$. The total number $n=\sum_{i=1}^{m} n_{i}$ is called the length of $s$ and it is denoted by $\ln (s)$. A subsemigroup $S$ of $S_{G}$ is called stable if there is an element $s \in S$ (called a stabilizing element of $S$ ) such that $s_{1} \cdot s=s_{2} \cdot s$ for any $s_{1}, s_{2} \in S$ such that $\alpha\left(s_{1}\right)=\alpha\left(s_{2}\right)$ and $\tau\left(s_{1}\right)=\tau\left(s_{2}\right)$.

For an element $s=x_{g_{1}} \cdot \ldots \cdot x_{g_{n}} \in S_{O}$ one can associate a subgroup $G_{s}=\left\langle g_{1}, \ldots, g_{n}\right\rangle$ of $G$ generated by the elements $g_{1}, \ldots, g_{n}$. For each two (not necessary proper) subgroups $H$ and $\Gamma$ of $G$ one can define subsemigroups $S_{O}^{H}=\left\{s \in S(G, O) \mid G_{s}=H\right\}$ and $S_{O, \Gamma}=\{s \in S(G, O) \mid \alpha(s) \in \Gamma\}$. If $H$ and $\Gamma$ are normal subgroups of $G$, then $S_{O, \Gamma}$ and $S_{O}^{H}$ are semigroups over $G$. By definition, $S_{O, \Gamma}^{H}=S_{O, \Gamma} \cap S_{O}^{H}$.

Let $\mathcal{S}_{d}$ be the symmetric group acting on the set $I_{d}=\{1, \ldots, d\}$ and $T_{d} \subset \mathcal{S}_{d}$ be the subset of transpositions. The semigroup $S_{\mathcal{S}_{d}}$ is denoted by $\Sigma_{d}$. By Theorem 2.3. in [1], the element

$$
h=\left(\prod_{i=1}^{d-1} x_{(i, i+1)}\right)^{3}
$$

is a stabilizing element of $\Sigma_{d}$, where $(i, i+1) \in T_{d}$ is a transposition permuting the elements $i$ and $i+1$ of $I_{d}$.

The aim of this article is to generalize this result to the case of almost all odd elements of the symmetric group $\mathcal{S}_{d}$. More precisely, let $C=C_{\sigma}$ be the conjugacy class of a permutation $\sigma \in \mathcal{S}_{d}$. As is known, if $\sigma$ is an odd permutation, then the elements of $C$ generate the group $\mathcal{S}_{d}$ and, in particular, any transposition $(i, j) \in \mathcal{S}_{d}$ is a product of some permutations belonging to $C$. Denote by $m_{C}$ the minimal number of permutations of $C$ needed to express $(1,2)$ as a product of permutations of $C$ and fix one of the such expressions:

$$
(1,2)=\sigma_{1} \ldots \sigma_{m_{C}}, \quad \sigma_{i} \in C .
$$

Denote by $n_{C}$ the order of $\sigma \in C$, by $k_{C}=|C|$ the number of elements of $C$, and by $f_{C}$ the number of elements of $I_{d}$ fixed under the action of $\sigma \in C$ on $I_{d}$.

Theorem 1. Let $C$ be the conjugacy class of an odd permutation $\sigma \in \mathcal{S}_{d}$. If $f_{C} \geqslant 2$ then there is a constant

$$
N=N_{C}<3^{d-3}(2 d-1)(d-1) m_{C}+n_{C} k_{C}+1
$$

such that any element $s=\widetilde{s} \cdot \bar{s} \in \Sigma_{d}^{\mathcal{S}_{d}}$, where $\bar{s} \in S_{C}$, is uniquely defined by $\tau(s)$ and $\alpha(s)$ if $\ln (\bar{s}) \geqslant N$.

Corollary 1. Let an equipped symmetric group $\left(\mathcal{S}_{d}, O\right)$ be such that the set $O$ contains a conjugacy class $C$ of an odd permutation $\sigma, f_{C} \geqslant 2$. Then $S_{O}=S\left(\mathcal{S}_{d}, O\right)$ is a stable semigroup. 
Note that in general case the constant $N_{C}$, existence of which is claimed in Theorem 1, is greater than 1. For example, as it was shown in [2], this is the case when $C$ is the conjugacy class of the permutation $\sigma=(1,2)(3,4,5) \in \mathcal{S}_{8}$.

The proof of Theorem 1] is similar to the proof of Theorem 2.3 in [1] and it is based on the following theorem.

Theorem 2. Let $C$ be the conjugacy class of an odd permutation $\sigma \in \mathcal{S}_{d}$ and an element $\bar{s}_{\left(i_{1}, i_{2}\right)} \in S_{C}$ be such that

(i) $\alpha\left(\bar{s}_{\left(i_{1}, i_{2}\right)}\right)=\left(i_{1}, i_{2}\right)$,

(ii) there are $i_{3}, i_{4} \in I_{d} \backslash\left\{i_{1}, i_{2}\right\}$ such that $\rho\left(\left(i_{3}, i_{4}\right)\right)\left(\bar{s}_{\left(i_{1}, i_{2}\right)}\right)=\bar{s}_{\left(i_{1}, i_{2}\right)}$.

Then there is an embedding over $\mathcal{S}_{d}$ of the semigroup $S_{T_{d}}$ in $S_{C}$.

Let $\operatorname{HUR}_{d, b}\left(\mathbb{P}^{1}\right)$ (resp., $\operatorname{HUR}_{d, b}^{G}\left(\mathbb{P}^{1}\right)$ ) be the Hurwitz space of ramified degree $d$ coverings of the projective line $\mathbb{P}^{1}$ (defined over $\mathbb{C}$ ) branched over $b$ points (and resp., with Galois group $G$ ). In [1], it was shown that the irreducible components of $\operatorname{HUR}_{d, b}\left(\mathbb{P}^{1}\right)$ are in one to one correspondence with the orbits of the action of $\mathcal{S}_{d}$ by simultaneous conjugations on $\Sigma_{d, \mathbf{1}, \mathbf{b}}=\left\{s \in \Sigma_{d, \mathbf{1}} \mid \ln (s)=b\right\}$ (that is, of the action defined by the homomorphism $\rho$ ) and if $G=\mathcal{S}_{d}$, then the irreducible components of $\operatorname{HUR}_{d, b}^{\mathcal{S}_{d}}\left(\mathbb{P}^{1}\right)$ are in one to one correspondence with the elements of $\Sigma_{d, \mathbf{1}}^{\mathcal{S}_{d}}$ of length equal to $b$. If an irreducible component of $\operatorname{HUR}_{d, b}^{\mathcal{S}_{d}}\left(\mathbb{P}^{1}\right)$ corresponds to an element $s \in \Sigma_{d, \mathbf{1}}^{\mathcal{S}_{d}}$, then we call $\tau(s)$ monodromy factorization type of the coverings belonging to this component. Denote by $\operatorname{HUR}_{d, t}^{\mathcal{S}_{d}}\left(\mathbb{P}^{1}\right)$ the union of irreducible components corresponding to the elements $s \in \Sigma_{d, 1}^{\mathcal{S}_{d}}$ with $\tau(s)=t$.

As a corollary of Theorem 1 we obtain

Theorem 3. Let $C$ be the conjugacy class of an odd permutation $\sigma \in \mathcal{S}_{d}$ such that $f_{C} \geqslant 2$. If the monodromy factorization type $t$ contains more than $N_{C}$ factors belonging to $C$, where $N_{C}$ is defined in Theorem 1 , then the space $\operatorname{HUR}_{d, t}^{\mathcal{S}_{d}}\left(\mathbb{P}^{1}\right)$ is irreducible.

\section{Proof of Theorem 2}

Without loss of generality, we can assume that $\left(i_{1}, i_{2}\right)=(1,2)$ and $\left(i_{3}, i_{4}\right)=(3,4)$.

For each $(i, j) \in T_{d}$ let us choose an element $\sigma_{i, j} \in \mathcal{S}_{d}$ such that $(i, j)=\sigma_{i, j}(1,2) \sigma_{i, j}^{-1}$ and put

$$
c=\bar{s}_{(1,2)}^{2} \cdot \bar{s}_{(2,3)}^{2} \cdot \ldots \cdot \bar{s}_{(d-1, d)}^{2},
$$

where $\bar{s}_{(i, j)}=\rho\left(\sigma_{i, j}\right)\left(\bar{s}_{(1,2)}\right)$.

Obviously, we have $\alpha\left(\bar{s}_{(i, j)}\right)=(i, j)$ and $\alpha(c)=\mathbf{1}$. Since the transpositions $(1,2), \ldots,(d-1, d)$ generate the group $\mathcal{S}_{d}$, then $c \in S_{C, \mathbf{1}}^{\mathcal{S}_{d}}$. Therefore, by Proposition 1.1 (2) in [1], the element $c$ is fixed under the conjugation action of $\mathcal{S}_{d}$ on $S_{C}$.

For $k \geqslant 4$ let us denote by $Z_{k} \simeq \mathcal{S}_{2} \times \mathcal{S}_{k-2}$ a subgroup of $\mathcal{S}_{d}$ generated by transpositions $(1,2)$ and $(i, j), 3 \leqslant i<j \leqslant k$. Note that $Z_{d}$ is the centralizer in $\mathcal{S}_{d}$ of the transposition $(1,2)$. 
Claim 1. There is an element $z_{(1,2)} \in S_{C}$ such that $\alpha\left(z_{(1,2)}\right)=(1,2)$ and $\rho(\sigma)\left(z_{(1,2)}\right)=$ $z_{(1,2)}$ for each $\sigma \in Z_{d}$

Proof. By induction in $k$, let us show that there is an element $y_{(1,2), k} \in S_{C}^{\mathcal{S}_{d}}$ such that $\alpha\left(y_{(1,2), k}\right)=(1,2)$ and $\rho(\sigma)\left(y_{(1,2), k}\right)=y_{(1,2), k}$ for each $\sigma \in Z_{k}$. Then $z_{(1,2)}=y_{(1,2), d}$ is a desired element.

Put $y_{(1,2), 4}=\bar{s}_{(1,2)} \cdot c$. If we move the first factor $\bar{s}_{(1,2)}$ to the right, then we obtain

$$
\begin{aligned}
y_{(1,2), 4}= & \bar{s}_{(1,2)} \cdot \bar{s}_{(1,2)} \cdot \bar{s}_{(1,2)} \cdot \bar{s}_{(2,3)}^{2} \cdot \ldots \cdot \bar{s}_{(d-1, d)}^{2}= \\
& \rho((1,2))\left(\bar{s}_{(1,2)}\right) \cdot \bar{s}_{(1,2)} \cdot \bar{s}_{(1,2)} \cdot \bar{s}_{(2,3)}^{2} \cdot \ldots \cdot \bar{s}_{(d-1, d)}^{2}= \\
& \rho((1,2))\left(\bar{s}_{(1,2)}\right) \cdot c=\rho((1,2))\left(\bar{s}_{(1,2)}\right) \cdot \rho((1,2))(c)= \\
& \rho((1,2))\left(\bar{s}_{(1,2)} \cdot c\right)=\rho((1,2))\left(y_{(1,2), 4}\right),
\end{aligned}
$$

since $c$ is fixed under the conjugation action of $\mathcal{S}_{d}$.

Similarly, by assumption of Theorem 2 ,

$\rho((3,4))\left(y_{(1,2), 4}\right)=\rho((3,4))\left(\bar{s}_{(1,2)} \cdot c\right)=\rho((3,4))\left(\bar{s}_{(1,2)}\right) \cdot \rho((3,4))(c)=\bar{s}_{(1,2)} \cdot c=y_{(1,2), 4}$ and hence $\rho(\sigma)\left(y_{(1,2), 4}\right)=y_{(1,2), 4}$ for all $\sigma \in Z_{4}$.

Assume that for some $k \geqslant 4, k<d$, we constructed an element $y_{(1,2), k} \in S_{C}^{\mathcal{S}_{d}}$ such that $\alpha\left(y_{(1,2), k}\right)=(1,2)$ and $\rho(\sigma)\left(y_{(1,2), k}\right)=y_{(1,2), k}$ for all $\sigma \in Z_{k}$. Consider an element $y_{(1,2), k}^{\prime}=\rho((k, k+1))\left(y_{(1,2), k}\right)$. Obviously, $y_{(1,2), k}^{\prime}$ belongs to $S_{C}^{\mathcal{S}_{d}}$ and it is easy to see that $\alpha\left(y_{(1,2), k}^{\prime}\right)=(1,2)$. Hence, the element $y_{(1,2), k} \cdot y_{(1,2), k}^{\prime}$ belongs to $S_{C, \mathbf{1}}^{\mathcal{S}_{d}}$ and therefore it is fixed under the conjugation action of $\mathcal{S}_{d}$. Besides, the element $y_{(1,2), k}^{\prime}$ is fixed under the action of the group $Z_{k}^{\prime}$ generated by transpositions $(i, j) \in Z_{k+1}$, $i, j \neq k$. Indeed, if $(i, j) \in Z_{k}^{\prime}$ and $i, j \neq k+1$, then

$$
\begin{aligned}
& \rho((i, j))\left(y_{(1,2), k}^{\prime}\right)=\rho((i, j))\left(\rho((k, k+1))\left(y_{(1,2), k}\right)\right)= \\
& \rho((i, j)(k, k+1))\left(y_{(1,2), k}\right)=\rho((k, k+1)(i, j))\left(y_{(1,2), k}\right)= \\
& \rho((k, k+1))\left(\rho((i, j))\left(y_{(1,2), k}\right)\right)=\rho((k, k+1))\left(y_{(1,2), k}\right)=y_{(1,2), k}^{\prime} .
\end{aligned}
$$

If $(i, k+1) \in Z_{k}^{\prime}$, then

$$
\begin{aligned}
& \rho((i, k+1))\left(y_{(1,2), k}^{\prime}\right)=\rho((i, k+1))\left(\rho((k, k+1))\left(y_{(1,2), k}\right)\right)= \\
& \rho((i, k+1)(k, k+1))\left(y_{(1,2), k}\right)=\rho((k, k+1)(i, k))\left(y_{(1,2), k}\right)= \\
& \rho((k, k+1))\left(\rho((i, k))\left(y_{(1,2), k}\right)=\rho((k, k+1))\left(y_{(1,2), k}\right)=y_{(1,2), k}^{\prime},\right.
\end{aligned}
$$

since $(i, k) \in Z_{k}$.

Moreover, the elements $y_{(1,2), k}, y_{(1,2), k}^{\prime}$ commute. Indeed,

$$
\begin{aligned}
& y_{(1,2), k}^{\prime} \cdot y_{(1,2), k}=\rho\left(\alpha\left(y_{(1,2), k}^{\prime}\right)\right)\left(y_{(1,2), k}\right) \cdot y_{(1,2), k}^{\prime}= \\
& \rho((1,2))\left(y_{(1,2), k}\right) \cdot y_{(1,2), k}^{\prime}=y_{(1,2), k} \cdot y_{(1,2), k}^{\prime} .
\end{aligned}
$$

Put $y_{(1,2), k+1}:=y_{(1,2), k}^{2} \cdot y_{(1,2), k}^{\prime}$. It is easy to see that $y_{(1,2), k+1} \in S_{C}^{\mathcal{S}_{d}}$ and $\alpha\left(y_{(1,2), k+1}\right)=$ $(1,2)$. Let us show that $\rho(\sigma)\left(y_{(1,2), k+1}\right)=y_{(1,2), k+1}$ for each $\sigma \in Z_{k+1}$. First of all note that the group $Z_{k+1}$ is generated by the elements of the groups $Z_{k}$ and $Z_{k}^{\prime}$. 
For each $\sigma \in Z_{k}$ we have

$$
\begin{aligned}
& \rho(\sigma)\left(y_{(1,2), k+1}\right)=\rho(\sigma)\left(y_{(1,2), k} \cdot y_{(1,2), k} \cdot y_{(1,2), k}^{\prime}\right)= \\
& \rho(\sigma)\left(y_{(1,2), k}\right) \cdot \rho(\sigma)\left(y_{(1,2), k} \cdot y_{(1,2), k}^{\prime}\right)=y_{(1,2), k} \cdot y_{(1,2), k} \cdot y_{(1,2), k}^{\prime},
\end{aligned}
$$

since the element $y_{(1,2), k} \cdot y_{(1,2), k}^{\prime} \in S_{C, \mathbf{1}}^{\mathcal{S}_{d}}$ is fixed under the conjugation action of $\mathcal{S}_{d}$.

Similarly, for each $\sigma \in Z_{k}^{\prime}$ we have

$$
\begin{aligned}
& \rho(\sigma)\left(y_{(1,2), k+1}\right)=\rho(\sigma)\left(y_{(1,2), k}^{2} \cdot y_{(1,2), k}^{\prime}\right)= \\
& \rho(\sigma)\left(y_{(1,2), k}^{2}\right) \cdot \rho(\sigma)\left(y_{(1,2), k}^{\prime}\right)=y_{(1,2), k}^{2} \cdot y_{(1,2), k}^{\prime}=y_{(1,2), k+1},
\end{aligned}
$$

since the element $y_{(1,2), k} \cdot y_{(1,2), k} \in S_{C, \mathbf{1}}^{\mathcal{S}_{d}}$ is fixed under the conjugation action of $\mathcal{S}_{d}$. Claim 1 is proved.

Consider the orbit $X_{T_{C, d}}$ of the element $z_{(1,2)}$ under the conjugation action of $\mathcal{S}_{d}$ on the semigroup $S_{C}$, where $z_{(1,2)}$ is the element constructed in the proof of Claim 1 with the help of the element $\bar{s}_{(1,2)}$.

Claim 2. The map $\bar{\alpha}: X_{T_{C, d}} \rightarrow X_{T_{d}}=\left\{x_{(i, j)} \mid(i, j) \in T_{d}\right\}$ given by $\bar{\alpha}\left(\rho(\sigma)\left(z_{(1,2)}\right)\right)=$ $x_{\sigma(1,2) \sigma^{-1}}$ is one-to-one correspondence.

Proof. The map $\bar{\alpha}: X_{T_{C, d}} \rightarrow X_{T_{d}}$ is surjective, since for each $(i, j) \in T_{d}$ there is $\sigma \in \mathcal{S}_{d}$ such that $(i, j)=\sigma(1,2) \sigma^{-1}$ and for this $\sigma$ we have

$$
\begin{gathered}
\alpha\left(\rho(\sigma)\left(z_{(1,2)}\right)\right)=\sigma(1,2) \sigma^{-1}=(i, j), \\
\alpha\left(\bar{\alpha}\left(\rho(\sigma)\left(z_{(1,2)}\right)\right)\right)=\alpha\left(x_{\sigma(1,2) \sigma^{-1}}\right)=\sigma(1,2) \sigma^{-1}=(i, j) .
\end{gathered}
$$

The order of the group $Z_{d}$ is equal to $2(d-2)$ !. Therefore, by Claim 1, the number $\left|X_{T_{C, d}}\right|$ of the elements of $X_{T_{C, d}}$ is not more than $\frac{d !}{2(d-2) !}=\frac{d(d-1)}{2}=\left|T_{d}\right|$ and hence $\bar{\alpha}: X_{T_{C, d}} \rightarrow X_{T_{d}}$ is one-to-one.

Denote by $z_{(i, j)}$ an element $z \in X_{T_{C, d}}$ such that $\alpha(z)=(i, j)$ and by $S_{T_{C, d}}$ a subsemigroup of $S_{C}$ generated by the elements $z_{(i, j)}, 1 \leqslant i, j \leqslant d, i \neq j$.

Claim 3. The subsemigroup $S_{T_{C, d}}$ of $S_{C}$ is a semigroup over $\mathcal{S}_{d}$ whose generators $z_{(i, j)}, 1 \leqslant i, j \leqslant d, i \neq j$, are subjected to the relations

$$
\begin{aligned}
& z_{(i, j)}=z_{(j, i)} \text { for all }\{i, j\}_{\text {ord }} \subset I_{d} \\
& z_{\left(i_{1}, i_{2}\right)} \cdot z_{\left(i_{1}, i_{3}\right)}=z_{\left(i_{2}, i_{3}\right)} \cdot z_{\left(i_{1}, i_{2}\right)}=z_{\left(i_{1}, i_{3}\right)} \cdot z_{\left(i_{2}, i_{3}\right)} \text { for all }\left\{i_{1}, i_{2}, i_{3}\right\}_{\text {ord }} \subset I_{d} \\
& z_{\left(i_{1}, i_{2}\right)} \cdot z_{\left(i_{3}, i_{4}\right)}=z_{\left(i_{3}, i_{4}\right)} \cdot z_{\left(i_{1}, i_{2}\right)} \text { for all }\left\{i_{1}, i_{2}, i_{3}, i_{4}\right\}_{\text {ord }} \subset I_{d} .
\end{aligned}
$$

Proof. Evident.

For $s \in S_{T_{C, d}}$, where $s$ is a product of $n$ generators $z_{(i, j)}$ of $S_{T_{C, d}}$, we define the $T$-length of $s$ as $\ln _{T}(s)=n$. Denote by $\bar{\alpha}^{-1}$ the map inverse to $\bar{\alpha}$. By Claim 3 , the map $\bar{\alpha}^{-1}: X_{T_{d}} \rightarrow X_{T_{C, d}}$ can be extended to a homomorphism $\bar{\alpha}^{-1}: S_{T_{d}} \rightarrow$ 
$S_{T_{C, d}}$ of semigroups over $\mathcal{S}_{d}$. Note that $\ln (s)=\ln _{T}\left(\bar{\alpha}^{-1}(s)\right)$ for $s \in S_{T_{d}}$. Define a subsemigroup $S_{T_{C, d}}^{\mathcal{S}_{d}, T}$ of $S_{T_{C, d}}$ as follows: $S_{T_{C, d}}^{\mathcal{S}_{d}, T}=\bar{\alpha}^{-1}\left(S_{T_{d}}^{\mathcal{S}_{d}}\right)$.

Now, Theorem 2 follows from

Claim 4. The homomorphism $\bar{\alpha}^{-1}: S_{T_{d}} \rightarrow S_{T_{C, d}}$ of semigroups over $\mathcal{S}_{d}$ is an isomorphism and, consequently, all statements of subsections 2.2, 2.3, and Lemma 2.9 in [1] remain true if we substitute $x_{(i, j)} \in S_{T_{d}}$ by $z_{(i, j)} \in S_{T_{C, d}}$, change the length of elements by $T$-length, and change $S_{T_{d}}^{\mathcal{S}_{d}}$ by $S_{T_{C, d}}^{\mathcal{S}_{d}, T}$.

Proof. Evident.

\section{Proof of Theorem 1}

Let us consider an element $\bar{s}_{(1,2)}=x_{\sigma_{1}} \cdot \ldots \cdot x_{\sigma_{m_{C}}}$, where $\sigma_{1}, \ldots, \sigma_{m_{C}} \in C$ are the factors in factorization (3).

If $f_{C} \geqslant 2$ then we can and will assume that all $\sigma_{i}$ entering into factorization (3) belong to the subgroup $\mathcal{S}_{d}^{\{3,4\}} \simeq \mathcal{S}_{d-2}$ of $\mathcal{S}_{d}$ the elements of which leave fixed the elements $3,4 \in I_{d}$. Therefore the element $\bar{s}_{(1,2)}=x_{\sigma_{1}} \cdot \ldots \cdot x_{\sigma_{m_{C}}}$ satisfies all conditions of Theorem 2 and, consequently, the elements $z_{(i, j)}$, constructed in section 1 with the help of $\bar{s}_{(1,2)}=x_{\sigma_{1}} \cdot \ldots \cdot x_{\sigma_{m_{C}}}$, generate in $S_{C}$ a semisubgroup isomorphic to $S_{T_{d}}$ over the group $\mathcal{S}_{d}$.

Note that the length of the element $z_{(1,2)}$, constructed in the proof of Claim 1, is equal to $\ln \left(z_{(1,2)}\right)=3^{d-4}(2 d-1) m_{C}$ if we start from the element $\bar{s}_{(1,2)}=x_{\sigma_{1}} \cdot \ldots \cdot x_{\sigma_{m_{C}}}$, where $\sigma_{1}, \ldots, \sigma_{m_{C}}$ are the factors of expression (3).

Denote by

$$
h_{C}=\left(z_{(1,2)} \cdot z_{(2,3)} \cdot \ldots \cdot z_{(d-1, d)}\right)^{3} .
$$

Rewrite $h_{C}$ as a product

$$
h_{C}=x_{\sigma_{1}} \cdot \ldots \cdot x_{\sigma_{L}}, \quad \sigma_{i} \in C \text { for } i=1, \ldots, L .
$$

It is easy to see that

$$
\ln \left(h_{C}\right)=3^{d-3}(2 d-1)(d-1) m_{C}:=L .
$$

To prove Theorem 1, we need the following

Claim 5. Under the conditions of Theorem 11, let an element $s=\widetilde{s} \cdot \bar{s} \in \Sigma_{d}^{\mathcal{S}_{d}}$ be such that $\bar{s} \in S_{C}$ of length $\ln (\bar{s}):=M \geqslant 3^{d-3}(2 d-1)(d-1) m_{C}+n_{C} k_{C}$. Then the element $s$ can be represented as $s=\widetilde{s}^{\prime} \cdot h_{C}$.

Proof. Let

$$
\bar{s}=x_{\sigma_{1}} \cdot \ldots \cdot x_{\sigma_{M}},
$$

$\sigma_{i} \in C$. Since $M=\ln (\bar{s}) \geqslant 3^{d-3}(2 d-1)(d-1) m_{C}+n_{C} k_{C}>n_{C} k_{C}$, then for some $\sigma \in C$ there are at least $n_{C}+1$ factors entering into factorization (5) equal to $\sigma$. 
Therefore $\bar{s}$ can be written in the form: $\bar{s}=\bar{s}^{\prime} \cdot x_{\sigma}^{n_{C}}$, where $\bar{s}^{\prime} \in S_{C}$ is such that $\widetilde{s} \cdot \bar{s}^{\prime} \in \Sigma_{d}^{\mathcal{S}_{d}}$. By Lemma 1.1 in [1], we have

$$
s=\widetilde{s} \cdot \bar{s}^{\prime} \cdot x_{\sigma}^{n_{C}}=\widetilde{s} \cdot \bar{s}^{\prime} \cdot x_{\sigma_{L}}^{n_{C}}=\widetilde{s} \cdot \bar{s}_{L} \cdot x_{\sigma_{L}},
$$

where $\bar{s}_{L}=\bar{s}^{\prime} \cdot x_{\sigma_{L}}^{n_{C}-1}$. Note that $\widetilde{s} \cdot \bar{s}_{L} \in \Sigma_{d}^{\mathcal{S}_{d}}$ and $\ln \left(\bar{s}_{L}\right)>n_{C} k_{C}$. Therefore, by the same arguments, the element $\widetilde{s}_{s} \cdot \bar{s}_{L}$ can be written in the form: $\widetilde{s}_{\bar{s}}=\widetilde{s}_{L} \cdot \bar{s}_{L}^{\prime} \cdot x_{\sigma_{L-1}}^{n_{C}-1} \cdot x_{\sigma_{L-1}}$. Put $\bar{s}_{L-1}=\bar{s}_{L}^{\prime} \cdot x_{\sigma_{L-1}}^{n_{C}-1}$. Repeating the same arguments for $\widetilde{s} \cdot \bar{s}_{L-1}$ we obtain that $\widetilde{s} \cdot \bar{s}_{L-1}=\widetilde{s} \cdot \bar{s}_{L-2} \cdot x_{\sigma_{L-1}}$, and so on. Finally, on the $L$ th step we obtain that

$$
s=\widetilde{s} \cdot \bar{s}=\widetilde{s} \cdot \bar{s}_{0} \cdot\left(x_{\sigma_{1}} \cdot \ldots \cdot x_{\sigma_{L}}\right)=\widetilde{s} \cdot \bar{s}_{0} \cdot h_{C} .
$$

Now to complete the proof of Theorem 1, recall that the proof of Theorem 3.2 in [1] consists of two parts. In the first part of the proof, for any element $s=\widetilde{s} \cdot \bar{s} \in \Sigma_{d}^{\mathcal{S}_{d}}$, where $\bar{s} \in S_{T_{d}}$ has the length $\ln (\bar{s}) \geqslant 3(d-1)$, it was proved the existence of another

factorization $s=\widetilde{s}_{1} \cdot \bar{s}_{1}$ such that $\bar{s}_{1} \in S_{T_{d}}^{\mathcal{S}_{d}}$ with $\ln \left(\bar{s}_{1}\right)=3(d-1)$. In this case the element $\bar{s}_{1}$ is uniquely determined by its product $\alpha\left(\bar{s}_{1}\right)=\alpha\left(\widetilde{s}_{1}\right)^{-1} \alpha(s)$. In the second part of the proof of Theorem 3.2 in [1] it was proved that for a such factorization $s=\widetilde{s}_{1} \cdot \bar{s}_{1}$ there is another factorization $s=\widetilde{s}_{2} \cdot \bar{s}_{2}$, where again $\bar{s}_{2} \in S_{T_{d}}^{\mathcal{S}_{d}}$ has the length $\ln \left(\bar{s}_{2}\right)=3(d-1)$ and $\widetilde{s}_{2}$ is uniquely determined by the type $\tau\left(\widetilde{s}_{1}\right)$. The proof of the last statement used only properties of the semigroup $S_{T_{d}}$ and relations (11) in the factorization semigroups. Therefore, by Claims 4 and 5 , the end of the proof of Theorem 1 coincides with the second part of the proof of Theorem 3.2 in [1].

\section{REFERENCES}

[1] Vik.S. Kulikov: Factorization semigroups and irreducible components of Hurwitz space. arXiv:1003.2953v1 (to appear in Izv. Math.).

[2] B. Wajnryb: Orbits of Hurwitz action for coverings of a sphere with two special fibres. Indag. Math. (N.S.), vol. 7 (1996), no. 4, $549-558$.

Steklov Mathematical Institute

E-mail address: kulikov@mi.ras.ru 


\title{
FACTORIZATION SEMIGROUPS AND IRREDUCIBLE COMPONENTS OF HURWITZ SPACE. II
}

\author{
VIK.S. KULIKOV
}

\begin{abstract}
This article is a continuation of the article [1]. Let $\operatorname{HUR}_{d, t}^{\mathcal{S}_{d}}\left(\mathbb{P}^{1}\right)$ be the Hurwitz space of degree $d$ coverings of the projective line $\mathbb{P}^{1}$ with Galois group $\mathcal{S}_{d}$ and having fixed monodromy type $t$ consisting of a collection of local monodromy types (that is, a collection of conjugacy classes of permutations $\sigma$ of the symmetric group $\mathcal{S}_{d}$ acting on the set $\left.I_{d}=\{1, \ldots, d\}\right)$. We prove that if the type $t$ contains big enough number of local monodromies belonging to the conjugacy class $C$ of an odd permutation $\sigma$ which leaves fixed $f_{C} \geqslant 2$ elements of $I_{d}$, then the Hurwitz space $\operatorname{HUR}_{d, t}^{\mathcal{S}_{d}}\left(\mathbb{P}^{1}\right)$ is irreducible.
\end{abstract}

\section{INTRODUCTION}

This article is a continuation of article [1]. To formulate the results of presented article, let us recall main definitions and notations used in [1]. A collection $(S, G, \alpha, \rho)$, where $S$ is a semigroup, $G$ is a group, and $\alpha: S \rightarrow G, \rho: G \rightarrow \operatorname{Aut}(S)$ are homomorphisms, is called a semigroup $S$ over a group $G$ if for all $s_{1}, s_{2} \in S$ we have

$$
s_{1} \cdot s_{2}=\rho\left(\alpha\left(s_{1}\right)\right)\left(s_{2}\right) \cdot s_{1}=s_{2} \cdot \lambda\left(\alpha\left(s_{2}\right)\right)\left(s_{1}\right),
$$

where $\lambda(g)=\rho\left(g^{-1}\right)$. Let $\left(S_{1}, G, \alpha_{1}, \rho_{1}\right)$ and $\left(S_{2}, G, \alpha_{2}, \rho_{2}\right)$ be two semigroups over a group $G$. A homomorphism of semigroups $\varphi: S_{1} \rightarrow S_{2}$ is said to be defined over $G$ if $\alpha_{1}(s)=\alpha_{2}(\varphi(s))$ and $\rho_{2}(g)(\varphi(s))=\varphi\left(\rho_{1}(g)(s)\right)$ for all $s \in S_{1}$ and $g \in G$.

A pair $(G, O)$, where $O \subset G$ is a subset of a group $G$ invariant under the inner automorphisms, is called an equipped group. To each equipped group $(G, O)$ one can associate a semigroup $S_{O}=S(G, O)$ over $G$ (called the factorization semigroup of the elements of $G$ with factors in $O$ ) generated by the elements of the alphabet $X=X_{O}=\left\{x_{g} \mid g \in O\right\}$ being subject to the following relations:

$$
x_{g_{1}} \cdot x_{g_{2}}=x_{g_{2}} \cdot x_{g_{2}^{-1} g_{1} g_{2}}=x_{g_{1} g_{2} g_{1}^{-1}} \cdot x_{g_{1}}
$$

for each $x_{g_{1}}, x_{g_{2}} \in X$ and if $g_{2}=\mathbf{1}$ then $x_{g_{1}} \cdot x_{\mathbf{1}}=x_{g_{1}}$. The map $\alpha: X \rightarrow G$, given by $\alpha\left(x_{g}\right)=g$ for each $x_{g} \in X$, induces a homomorphism $\alpha: S_{O} \rightarrow G$ called the product homomorphism. The action (from the left) $\rho$ of the group $G$ on $S_{O}$ is defined by the action on the alphabet $X$ as follows:

$$
x_{a} \in X \mapsto \rho(g)\left(x_{a}\right)=x_{g a g^{-1}} \in X
$$

This research was partially supported by grants of NSh-4713.2010.1, RFBR 11-01-00185, and by AG Laboratory HSE, RF government grant, ag. 11.G34.31.0023. 
for each $g \in G$. Note that $\alpha(\rho(g)(s))=g \alpha(s) g^{-1}$ for all $s \in S_{O}$ and all $g \in G$.

Let $O \backslash\{\mathbf{1}\}=C_{1} \sqcup \cdots \sqcup C_{m}$ be the decomposition of the set $O$ into the disjoint union of conjugacy classes of elements of $G$. Each element $s=x_{g_{1}} \cdot \ldots \cdot x_{g_{n}} \in S_{O}$ defines an element $\tau(s)=n_{1} C_{1}+\cdots+n_{m} C_{m}$ of the free abelian semigroup generated by $C_{1}, \ldots, C_{m}(\tau(s)$ is called the type of $s)$, where $n_{i}$ is equal to the number of factors $x_{g_{j}}$ entering in the factorization $s=x_{g_{1}} \cdot \ldots \cdot x_{g_{n}}$ for which $g_{j} \in C_{i}$. The total number $n=\sum_{i=1}^{m} n_{i}$ is called the length of $s$ and it is denoted by $\ln (s)$. A subsemigroup $S$ of $S_{G}$ is called stable if there is an element $s \in S$ (called a stabilizing element of $S$ ) such that $s_{1} \cdot s=s_{2} \cdot s$ for any $s_{1}, s_{2} \in S$ such that $\alpha\left(s_{1}\right)=\alpha\left(s_{2}\right)$ and $\tau\left(s_{1}\right)=\tau\left(s_{2}\right)$.

For an element $s=x_{g_{1}} \cdot \ldots \cdot x_{g_{n}} \in S_{O}$ one can associate a subgroup $G_{s}=\left\langle g_{1}, \ldots, g_{n}\right\rangle$ of $G$ generated by the elements $g_{1}, \ldots, g_{n}$. For each two (not necessary proper) subgroups $H$ and $\Gamma$ of $G$ one can define subsemigroups $S_{O}^{H}=\left\{s \in S(G, O) \mid G_{s}=H\right\}$ and $S_{O, \Gamma}=\{s \in S(G, O) \mid \alpha(s) \in \Gamma\}$. If $H$ and $\Gamma$ are normal subgroups of $G$, then $S_{O, \Gamma}$ and $S_{O}^{H}$ are semigroups over $G$. By definition, $S_{O, \Gamma}^{H}=S_{O, \Gamma} \cap S_{O}^{H}$.

Let $\mathcal{S}_{d}$ be the symmetric group acting on the set $I_{d}=\{1, \ldots, d\}$ and $T_{d} \subset \mathcal{S}_{d}$ be the subset of transpositions. The semigroup $S_{\mathcal{S}_{d}}$ is denoted by $\Sigma_{d}$. By Theorem 2.3. in [1], the element

$$
h=\left(\prod_{i=1}^{d-1} x_{(i, i+1)}\right)^{3}
$$

is a stabilizing element of $\Sigma_{d}$, where $(i, i+1) \in T_{d}$ is a transposition permuting the elements $i$ and $i+1$ of $I_{d}$.

The aim of this article is to generalize this result to the case of almost all odd elements of the symmetric group $\mathcal{S}_{d}$. More precisely, let $C=C_{\sigma}$ be the conjugacy class of a permutation $\sigma \in \mathcal{S}_{d}$. Denote by $n_{C}$ the order of $\sigma \in C$, by $k_{C}=|C|$ the number of elements of $C$, and by $f_{C}$ the number of elements of $I_{d}$ fixed under the action of $\sigma \in C$ on $I_{d}$.

As is known, if $\sigma$ is an odd permutation, then the elements of $C$ generate the group $\mathcal{S}_{d}$ and, in particular, any transposition $(i, j) \in \mathcal{S}_{d}$ is a product of some permutations belonging to $C$. In the case $f_{C} \geqslant 2$, denote by $m_{C}$ the minimal number (counted with multiplicities) of permutations of $C \cap \mathcal{S}_{d-2}$ needed to express $(1,2)$ as a product of permutations of $C$ and fix one of the such expressions:

$$
(1,2)=\sigma_{1} \ldots \sigma_{m_{C}}, \quad \sigma_{i} \in C \cap \mathcal{S}_{d-2} .
$$

Theorem 1. Let $C$ be the conjugacy class of an odd permutation $\sigma \in \mathcal{S}_{d}$. If $f_{C} \geqslant 2$ then there is a constant

$$
N=N_{C}<3^{d-3}(2 d-1)(d-1) m_{C}+n_{C} k_{C}+1
$$

such that any element $s=\widetilde{s} \cdot \bar{s} \in \Sigma_{d}^{\mathcal{S}_{d}}$, where $\bar{s} \in S_{C}$, is uniquely defined by $\tau(s)$ and $\alpha(s)$ if $\ln (\bar{s}) \geqslant N$. 
Corollary 1. Let an equipped symmetric group $\left(\mathcal{S}_{d}, O\right)$ be such that the set $O$ contains a conjugacy class $C$ of an odd permutation $\sigma, f_{C} \geqslant 2$. Then $S_{O}=S\left(\mathcal{S}_{d}, O\right)$ is a stable semigroup.

Note that in general case the constant $N_{C}$, existence of which is claimed in Theorem 1, is greater than 1. For example, as it was shown in [2], this is the case when $C$ is the conjugacy class of the permutation $\sigma=(1,2)(3,4,5) \in \mathcal{S}_{8}$.

The proof of Theorem 1 is similar to the proof of Theorem 2.3 in [1] and it is based on the following theorem.

Theorem 2. Let $C$ be the conjugacy class of an odd permutation $\sigma \in \mathcal{S}_{d}$ and an element $\bar{s}_{\left(i_{1}, i_{2}\right)} \in S_{C}$ be such that

(i) $\alpha\left(\bar{s}_{\left(i_{1}, i_{2}\right)}\right)=\left(i_{1}, i_{2}\right)$,

(ii) there are $i_{3}, i_{4} \in I_{d} \backslash\left\{i_{1}, i_{2}\right\}$ such that $\rho\left(\left(i_{3}, i_{4}\right)\right)\left(\bar{s}_{\left(i_{1}, i_{2}\right)}\right)=\bar{s}_{\left(i_{1}, i_{2}\right)}$.

Then there is an embedding over $\mathcal{S}_{d}$ of the semigroup $S_{T_{d}}^{\mathcal{S}_{d}}$ in $S_{C}$.

Let $\operatorname{HUR}_{d, b}\left(\mathbb{P}^{1}\right)$ (resp., HUR $\mathrm{H}_{d, b}^{G}\left(\mathbb{P}^{1}\right)$ ) be the Hurwitz space of ramified degree $d$ coverings of the projective line $\mathbb{P}^{1}$ (defined over $\mathbb{C}$ ) branched over $b$ points (and resp., with Galois group $G$ ). In [1], it was shown that the irreducible components of $\operatorname{HUR}_{d, b}\left(\mathbb{P}^{1}\right)$ are in one to one correspondence with the orbits of the action of $\mathcal{S}_{d}$ by simultaneous conjugations on $\Sigma_{d, \mathbf{1}, \mathbf{b}}=\left\{s \in \Sigma_{d, \mathbf{1}} \mid \ln (s)=b\right\}$ (that is, of the action defined by the homomorphism $\rho$ ) and if $G=\mathcal{S}_{d}$, then the irreducible components of $\operatorname{HUR}_{d, b}^{\mathcal{S}_{d}}\left(\mathbb{P}^{1}\right)$ are in one to one correspondence with the elements of $\Sigma_{d, \mathbf{1}}^{\mathcal{S}_{d}}$ of length equal to $b$. If an irreducible component of $\operatorname{HUR}_{d, b}^{\mathcal{S}_{d}}\left(\mathbb{P}^{1}\right)$ corresponds to an element $s \in \Sigma_{d, \mathbf{1}}^{\mathcal{S}_{d}}$, then we call $\tau(s)$ monodromy factorization type of the coverings belonging to this component. Denote by $\operatorname{HUR}_{d, t}^{\mathcal{S}_{d}}\left(\mathbb{P}^{1}\right)$ the union of irreducible components corresponding to the elements $s \in \Sigma_{d, \mathbf{1}}^{\mathcal{S}_{d}}$ with $\tau(s)=t$.

As a corollary of Theorem 1 we obtain

Theorem 3. Let $C$ be the conjugacy class of an odd permutation $\sigma \in \mathcal{S}_{d}$ such that $f_{C} \geqslant 2$. If the monodromy factorization type $t$ contains more than $N_{C}$ factors belonging to $C$, where $N_{C}$ is defined in Theorem 1 , then the space $\operatorname{HUR}_{d, t}^{\mathcal{S}_{d}}\left(\mathbb{P}^{1}\right)$ is irreducible.

Note that fot Hurwitz spaces of $d$-sheeted coverings of the disc $\Delta=\{z \in \mathbb{C}|| z \mid \leqslant$ $1\}$ (respectively, of $d$-sheeted coverings of the affine line $\mathbb{C}^{1}$ ), a statement, similar to Theorem [3, is also true.

\section{Proof of Theorem 2}

Without loss of generality, we can assume that $\left(i_{1}, i_{2}\right)=(1,2)$ and $\left(i_{3}, i_{4}\right)=(3,4)$.

For each $(i, j) \in T_{d}$ let us choose an element $\sigma_{i, j} \in \mathcal{S}_{d}$ such that $(i, j)=\sigma_{i, j}(1,2) \sigma_{i, j}^{-1}$ and put

$$
c=\bar{s}_{(1,2)}^{2} \cdot \bar{s}_{(2,3)}^{2} \cdot \ldots \cdot \bar{s}_{(d-1, d)}^{2},
$$

where $\bar{s}_{(i, j)}=\rho\left(\sigma_{i, j}\right)\left(\bar{s}_{(1,2)}\right)$. 
Obviously, we have $\alpha\left(\bar{s}_{(i, j)}\right)=(i, j)$ and $\alpha(c)=\mathbf{1}$. Since the transpositions $(1,2), \ldots,(d-1, d)$ generate the group $\mathcal{S}_{d}$, then $c \in S_{C, \mathbf{1}}^{\mathcal{S}_{d}}$. Therefore, by Proposition 1.1 (2) in [1], the element $c$ is fixed under the conjugation action of $\mathcal{S}_{d}$ on $S_{C}$.

For $k \geqslant 4$ let us denote by $Z_{k} \simeq \mathcal{S}_{2} \times \mathcal{S}_{k-2}$ a subgroup of $\mathcal{S}_{d}$ generated by transpositions $(1,2)$ and $(i, j), 3 \leqslant i<j \leqslant k$. Note that $Z_{d}$ is the centralizer in $\mathcal{S}_{d}$ of the transposition $(1,2)$.

Claim 1. There is an element $z_{(1,2)} \in S_{C}$ such that $\alpha\left(z_{(1,2)}\right)=(1,2)$ and $\rho(\sigma)\left(z_{(1,2)}\right)=$ $z_{(1,2)}$ for each $\sigma \in Z_{d}$

Proof. By induction in $k$, let us show that there is an element $y_{(1,2), k} \in S_{C}^{\mathcal{S}_{d}}$ such that $\alpha\left(y_{(1,2), k}\right)=(1,2)$ and $\rho(\sigma)\left(y_{(1,2), k}\right)=y_{(1,2), k}$ for each $\sigma \in Z_{k}$. Then $z_{(1,2)}=y_{(1,2), d}$ is a desired element.

Put $y_{(1,2), 4}=\bar{s}_{(1,2)} \cdot c$. If we move the first factor $\bar{s}_{(1,2)}$ to the right, then we obtain

$$
\begin{aligned}
y_{(1,2), 4}= & \bar{s}_{(1,2)} \cdot \bar{s}_{(1,2)} \cdot \bar{s}_{(1,2)} \cdot \bar{s}_{(2,3)}^{2} \cdot \ldots \cdot \bar{s}_{(d-1, d)}^{2}= \\
& \rho((1,2))\left(\bar{s}_{(1,2)}\right) \cdot \bar{s}_{(1,2)} \cdot \bar{s}_{(1,2)} \cdot \bar{s}_{(2,3)}^{2} \cdot \ldots \cdot \bar{s}_{(d-1, d)}^{2}= \\
& \rho((1,2))\left(\bar{s}_{(1,2)}\right) \cdot c=\rho((1,2))\left(\bar{s}_{(1,2)}\right) \cdot \rho((1,2))(c)= \\
& \rho((1,2))\left(\bar{s}_{(1,2)} \cdot c\right)=\rho((1,2))\left(y_{(1,2), 4}\right),
\end{aligned}
$$

since $c$ is fixed under the conjugation action of $\mathcal{S}_{d}$.

Similarly, by assumption of Theorem 2 ,

$\rho((3,4))\left(y_{(1,2), 4}\right)=\rho((3,4))\left(\bar{s}_{(1,2)} \cdot c\right)=\rho((3,4))\left(\bar{s}_{(1,2)}\right) \cdot \rho((3,4))(c)=\bar{s}_{(1,2)} \cdot c=y_{(1,2), 4}$ and hence $\rho(\sigma)\left(y_{(1,2), 4}\right)=y_{(1,2), 4}$ for all $\sigma \in Z_{4}$.

Assume that for some $k \geqslant 4, k<d$, we constructed an element $y_{(1,2), k} \in S_{C}^{\mathcal{S}_{d}}$ such that $\alpha\left(y_{(1,2), k}\right)=(1,2)$ and $\rho(\sigma)\left(y_{(1,2), k}\right)=y_{(1,2), k}$ for all $\sigma \in Z_{k}$. Consider an element $y_{(1,2), k}^{\prime}=\rho((k, k+1))\left(y_{(1,2), k}\right)$. Obviously, $y_{(1,2), k}^{\prime}$ belongs to $S_{C}^{\mathcal{S}_{d}}$ and it is easy to see that $\alpha\left(y_{(1,2), k}^{\prime}\right)=(1,2)$. Hence, the element $y_{(1,2), k} \cdot y_{(1,2), k}^{\prime}$ belongs to $S_{C, \mathbf{1}}^{\mathcal{S}_{d}}$ and therefore it is fixed under the conjugation action of $\mathcal{S}_{d}$. Besides, the element $y_{(1,2), k}^{\prime}$ is fixed under the action of the group $Z_{k}^{\prime}$ generated by transpositions $(i, j) \in Z_{k+1}$, $i, j \neq k$. Indeed, if $(i, j) \in Z_{k}^{\prime}$ and $i, j \neq k+1$, then

$$
\begin{aligned}
& \rho((i, j))\left(y_{(1,2), k}^{\prime}\right)=\rho((i, j))\left(\rho((k, k+1))\left(y_{(1,2), k}\right)\right)= \\
& \rho((i, j)(k, k+1))\left(y_{(1,2), k}\right)=\rho((k, k+1)(i, j))\left(y_{(1,2), k}\right)= \\
& \rho((k, k+1))\left(\rho((i, j))\left(y_{(1,2), k}\right)\right)=\rho((k, k+1))\left(y_{(1,2), k}\right)=y_{(1,2), k}^{\prime} .
\end{aligned}
$$

If $(i, k+1) \in Z_{k}^{\prime}$, then

$$
\begin{aligned}
& \rho((i, k+1))\left(y_{(1,2), k}^{\prime}\right)=\rho((i, k+1))\left(\rho((k, k+1))\left(y_{(1,2), k}\right)\right)= \\
& \rho((i, k+1)(k, k+1))\left(y_{(1,2), k}\right)=\rho((k, k+1)(i, k))\left(y_{(1,2), k}\right)= \\
& \rho((k, k+1))\left(\rho((i, k))\left(y_{(1,2), k}\right)=\rho((k, k+1))\left(y_{(1,2), k}\right)=y_{(1,2), k}^{\prime},\right.
\end{aligned}
$$

since $(i, k) \in Z_{k}$. 
Moreover, the elements $y_{(1,2), k}, y_{(1,2), k}^{\prime}$ commute. Indeed,

$$
\begin{aligned}
& y_{(1,2), k}^{\prime} \cdot y_{(1,2), k}=\rho\left(\alpha\left(y_{(1,2), k}^{\prime}\right)\right)\left(y_{(1,2), k}\right) \cdot y_{(1,2), k}^{\prime}= \\
& \rho((1,2))\left(y_{(1,2), k}\right) \cdot y_{(1,2), k}^{\prime}=y_{(1,2), k} \cdot y_{(1,2), k}^{\prime} .
\end{aligned}
$$

Put $y_{(1,2), k+1}:=y_{(1,2), k}^{2} \cdot y_{(1,2), k}^{\prime}$. It is easy to see that $y_{(1,2), k+1} \in S_{C}^{\mathcal{S}_{d}}$ and $\alpha\left(y_{(1,2), k+1}\right)=$ $(1,2)$. Let us show that $\rho(\sigma)\left(y_{(1,2), k+1}\right)=y_{(1,2), k+1}$ for each $\sigma \in Z_{k+1}$. First of all note that the group $Z_{k+1}$ is generated by the elements of the groups $Z_{k}$ and $Z_{k}^{\prime}$.

For each $\sigma \in Z_{k}$ we have

$$
\begin{aligned}
& \rho(\sigma)\left(y_{(1,2), k+1}\right)=\rho(\sigma)\left(y_{(1,2), k} \cdot y_{(1,2), k} \cdot y_{(1,2), k}^{\prime}\right)= \\
& \rho(\sigma)\left(y_{(1,2), k}\right) \cdot \rho(\sigma)\left(y_{(1,2), k} \cdot y_{(1,2), k}^{\prime}\right)=y_{(1,2), k} \cdot y_{(1,2), k} \cdot y_{(1,2), k}^{\prime},
\end{aligned}
$$

since the element $y_{(1,2), k} \cdot y_{(1,2), k}^{\prime} \in S_{C, \mathbf{1}}^{\mathcal{S}_{d}}$ is fixed under the conjugation action of $\mathcal{S}_{d}$.

Similarly, for each $\sigma \in Z_{k}^{\prime}$ we have

$$
\begin{aligned}
& \rho(\sigma)\left(y_{(1,2), k+1}\right)=\rho(\sigma)\left(y_{(1,2), k}^{2} \cdot y_{(1,2), k}^{\prime}\right)= \\
& \rho(\sigma)\left(y_{(1,2), k}^{2}\right) \cdot \rho(\sigma)\left(y_{(1,2), k}^{\prime}\right)=y_{(1,2), k}^{2} \cdot y_{(1,2), k}^{\prime}=y_{(1,2), k+1},
\end{aligned}
$$

since the element $y_{(1,2), k} \cdot y_{(1,2), k} \in S_{C, 1}^{\mathcal{S}_{d}}$ is fixed under the conjugation action of $\mathcal{S}_{d}$. Claim 1 is proved.

Consider the orbit $X_{T_{C, d}}$ of the element $z_{(1,2)}$ under the conjugation action of $\mathcal{S}_{d}$ on the semigroup $S_{C}$, where $z_{(1,2)}$ is the element constructed in the proof of Claim 1 with the help of the element $\bar{s}_{(1,2)}$.

Claim 2. The map $\bar{\alpha}: X_{T_{C, d}} \rightarrow X_{T_{d}}=\left\{x_{(i, j)} \mid(i, j) \in T_{d}\right\}$ given by $\bar{\alpha}\left(\rho(\sigma)\left(z_{(1,2)}\right)\right)=$ $x_{\sigma(1,2) \sigma^{-1}}$ is one-to-one correspondence.

Proof. The map $\bar{\alpha}: X_{T_{C, d}} \rightarrow X_{T_{d}}$ is surjective, since for each $(i, j) \in T_{d}$ there is $\sigma \in \mathcal{S}_{d}$ such that $(i, j)=\sigma(1,2) \sigma^{-1}$ and for this $\sigma$ we have

$$
\begin{gathered}
\alpha\left(\rho(\sigma)\left(z_{(1,2)}\right)\right)=\sigma(1,2) \sigma^{-1}=(i, j), \\
\alpha\left(\bar{\alpha}\left(\rho(\sigma)\left(z_{(1,2)}\right)\right)\right)=\alpha\left(x_{\sigma(1,2) \sigma^{-1}}\right)=\sigma(1,2) \sigma^{-1}=(i, j) .
\end{gathered}
$$

The order of the group $Z_{d}$ is equal to $2(d-2)$ !. Therefore, by Claim 1, the number $\left|X_{T_{C, d}}\right|$ of the elements of $X_{T_{C, d}}$ is not more than $\frac{d !}{2(d-2) !}=\frac{d(d-1)}{2}=\left|T_{d}\right|$ and hence $\bar{\alpha}: X_{T_{C, d}} \rightarrow X_{T_{d}}$ is one-to-one.

Denote by $z_{(i, j)}$ an element $z \in X_{T_{C, d}}$ such that $\alpha(z)=(i, j)$ and by $S_{T_{C, d}}$ a subsemigroup of $S_{C}$ generated by the elements $z_{(i, j)}, 1 \leqslant i, j \leqslant d, i \neq j$.

Claim 3. The subsemigroup $S_{T_{C, d}}$ of $S_{C}$ is a semigroup over $\mathcal{S}_{d}$ The elements $z_{(i, j)} \in$ $S_{T_{C, d}}, 1 \leqslant i, j \leqslant d, i \neq j$, satisfy the following relations

$$
\begin{aligned}
& z_{(i, j)}=z_{(j, i)} \text { for all }\{i, j\}_{\text {ord }} \subset I_{d} ; \\
& z_{\left(i_{1}, i_{2}\right)} \cdot z_{\left(i_{1}, i_{3}\right)}=z_{\left(i_{2}, i_{3}\right)} \cdot z_{\left(i_{1}, i_{2}\right)}=z_{\left(i_{1}, i_{3}\right)} \cdot z_{\left(i_{2}, i_{3}\right)} \text { for all }\left\{i_{1}, i_{2}, i_{3}\right\}_{\text {ord }} \subset I_{d} \\
& z_{\left(i_{1}, i_{2}\right)} \cdot z_{\left(i_{3}, i_{4}\right)}=z_{\left(i_{3}, i_{4}\right)} \cdot z_{\left(i_{1}, i_{2}\right)} \text { for all }\left\{i_{1}, i_{2}, i_{3}, i_{4}\right\}_{\text {ord }} \subset I_{d} .
\end{aligned}
$$


Proof. It directly follows from constructions of the elements $z_{(i, j)}$ and Claim 1.1 in [1].

Claim 4. The map $\bar{\alpha}^{-1}: X_{T_{d}} \rightarrow X_{T_{C, d}}$ is extended to surjective homomorphism $\bar{\alpha}^{-1}: S_{T_{d}} \rightarrow S_{T_{C, d}}$ of semigroups over group $\mathcal{S}_{d}$.

Proof. Note that if to substitute $x_{(i, j)}$ instead of $z_{(i, j)}$ in relations (44), then we obtain the defining relations in the semigroup $S_{T_{d}}$. Therefore, it follows from Claim 3 that $\bar{\alpha}^{-1}$ can be extended to a surjective homomorphism semigroups over group $\mathcal{S}_{d}$.

For $s \in S_{T_{C, d}}$, where $s$ is a product of $n$ generators $z_{(i, j)}$ of $S_{T_{C, d}}$, we define the $T$-length of $s$ as $\ln _{T}(s)=n$. We have $\ln (s)=\ln _{T}\left(\bar{\alpha}^{-1}(s)\right)$ for $s \in S_{T_{d}}$.

Note that it follows from Claim 4 that any statement in 11, in which it is claimed that an element of $S_{T_{d}}$ can be represented as the product of some generators $x_{i, j}$, is true for elements of $S_{T_{C, d}}$ if in the statement we change the elements $x_{(i, j)}$ by $z_{(i, j)}$ and change the lengths by $T$-lengths.

Define a subsemigroup $S_{T_{C, d}}^{\mathcal{S}_{d}, T}$ of $S_{T_{C, d}}$ as follows: $S_{T_{C, d}}^{\mathcal{S}_{d}, T}=\bar{\alpha}^{-1}\left(S_{T_{d}}^{\mathcal{S}_{d}}\right)$.

Theorem 2 follows from

Claim 5. The restriction of $\bar{\alpha}^{-1}: S_{T_{d}} \rightarrow S_{T_{C, d}}$ to $S_{T_{d}}^{\mathcal{S}_{d}}$,

$$
\bar{\alpha}^{-1}: S_{T_{d}}^{\mathcal{S}_{d}} \rightarrow S_{T_{C, d}}^{\mathcal{S}_{d}, T}
$$

is an isomorphism of semigroups over the group $\mathcal{S}_{d}$.

Proof. It follows from Theorem 2.1 in [1] that the homomorphism $\bar{\alpha}^{-1}: S_{T_{d}}^{\mathcal{S}_{d}} \rightarrow S_{T_{C, d}}^{\mathcal{S}_{d}, T}$ is injective.

Note also that Theorem 2.1 in [1] and Claim 5 imply directly the following

Corollary 2. The elements $s$ of the semigroup $S_{T_{C, d}}^{\mathcal{S}_{d}, T}$ are defined uniquely by $\alpha(s)$ and $\ln _{T}(s)$.

\section{Proof of Theorem 1}

Let us consider an element $\bar{s}_{(1,2)}=x_{\sigma_{1}} \cdot \ldots \cdot x_{\sigma_{m_{C}}}$, where $\sigma_{1}, \ldots, \sigma_{m_{C}} \in C$ are the factors in factorization (3).

If $f_{C} \geqslant 2$ then we can and will assume that all $\sigma_{i}$ entering into factorization (3) belong to the subgroup $\mathcal{S}_{d}^{\{3,4\}} \simeq \mathcal{S}_{d-2}$ of $\mathcal{S}_{d}$ the elements of which leave fixed the elements $3,4 \in I_{d}$. Therefore the element $\bar{s}_{(1,2)}=x_{\sigma_{1}} \cdot \ldots \cdot x_{\sigma_{m_{C}}}$ satisfies all conditions of Theorem 2 and, consequently, the elements $z_{(i, j)}$, constructed in section 1 with the help of $\bar{s}_{(1,2)}=x_{\sigma_{1}} \cdot \ldots \cdot x_{\sigma_{m_{C}}}$, define uniquely a semisubgroup $S_{T_{C, d}}^{\mathcal{S}_{d}, T}$ of $S_{C}$ isomorphic to $S_{T_{d}}$ over the group $\mathcal{S}_{d}$.

Note that the length of the element $z_{(1,2)}$, constructed in the proof of Claim 11, is equal to $\ln \left(z_{(1,2)}\right)=3^{d-4}(2 d-1) m_{C}$ if we start from the element $\bar{s}_{(1,2)}=x_{\sigma_{1}} \cdot \ldots \cdot x_{\sigma_{m_{C}}}$, where $\sigma_{1}, \ldots, \sigma_{m_{C}}$ are the factors of expression (3). 
Denote by

$$
h_{C}=\left(z_{(1,2)} \cdot z_{(2,3)} \cdot \ldots \cdot z_{(d-1, d)}\right)^{3} .
$$

Rewrite $h_{C}$ as a product

$$
h_{C}=x_{\sigma_{1}} \cdot \ldots \cdot x_{\sigma_{L}}, \quad \sigma_{i} \in C \text { for } i=1, \ldots, L .
$$

It is easy to see that

$$
\ln \left(h_{C}\right)=3^{d-3}(2 d-1)(d-1) m_{C}:=L .
$$

To prove Theorem 1, we need the following

Claim 6. Under the conditions of Theorem 1, let an element $s=\widetilde{s} \cdot \bar{s} \in \Sigma_{d}^{\mathcal{S}_{d}}$ be such that $\bar{s} \in S_{C}$ of length $\ln (\bar{s}):=M \geqslant 3^{d-3}(2 d-1)(d-1) m_{C}+n_{C} k_{C}$. Then the element $s$ can be represented as $s=\widetilde{s}^{\prime} \cdot h_{C}$.

Proof. Let

$$
\bar{s}=x_{\sigma_{1}} \cdot \ldots \cdot x_{\sigma_{M}}
$$

$\sigma_{i} \in C$. Since $M=\ln (\bar{s}) \geqslant 3^{d-3}(2 d-1)(d-1) m_{C}+n_{C} k_{C}>n_{C} k_{C}$, then for some $\sigma \in C$ there are at least $n_{C}+1$ factors entering into factorization (5) equal to $\sigma$. Therefore $\bar{s}$ can be written in the form: $\bar{s}=\bar{s}^{\prime} \cdot x_{\sigma}^{n_{C}}$, where $\bar{s}^{\prime} \in S_{C}$ is such that $\widetilde{s} \cdot \bar{s}^{\prime} \in \Sigma_{d}^{\mathcal{S}_{d}}$. By Lemma 1.1 in [1], we have

$$
s=\widetilde{s} \cdot \bar{s}^{\prime} \cdot x_{\sigma}^{n_{C}}=\widetilde{s} \cdot \bar{s}^{\prime} \cdot x_{\sigma_{L}}^{n_{C}}=\widetilde{s} \cdot \bar{s}_{L} \cdot x_{\sigma_{L}},
$$

where $\bar{s}_{L}=\bar{s}^{\prime} \cdot x_{\sigma_{L}}^{n_{C}-1}$. Note that $\widetilde{s} \cdot \bar{s}_{L} \in \Sigma_{d}^{\mathcal{S}_{d}}$ and $\ln \left(\bar{s}_{L}\right)>n_{C} k_{C}$. Therefore, by the same arguments, the element $\widetilde{s} \cdot \bar{s}_{L}$ can be written in the form: $\widetilde{s} \cdot \bar{s}_{L}=\widetilde{s} \cdot \bar{s}_{L}^{\prime} \cdot x_{\sigma_{L-1}}^{n_{C}-1} \cdot x_{\sigma_{L-1}}$. Put $\bar{s}_{L-1}=\bar{s}_{L}^{\prime} \cdot x_{\sigma_{L-1}}^{n_{C}-1}$. Repeating the same arguments for $\widetilde{s} \cdot \bar{s}_{L-1}$ we obtain that $\widetilde{s} \cdot \bar{s}_{L-1}=\widetilde{s} \cdot \bar{s}_{L-2} \cdot x_{\sigma_{L-1}}$, and so on. Finally, on the $L$ th step we obtain that

$$
s=\widetilde{s} \cdot \bar{s}=\widetilde{s} \cdot \bar{s}_{0} \cdot\left(x_{\sigma_{1}} \cdot \ldots \cdot x_{\sigma_{L}}\right)=\widetilde{s} \cdot \bar{s}_{0} \cdot h_{C} .
$$

Now to complete the proof of Theorem 1, recall that the proof of Theorem 3.2 in [1] consists of two parts. In the first part of the proof, for any element $s=\widetilde{s} \cdot \bar{s} \in \Sigma_{d}^{\mathcal{S}_{d}}$, where $\bar{s} \in S_{T_{d}}$ has the length $\ln (\bar{s}) \geqslant 3(d-1)$, it was proved the existence of another factorization $s=\widetilde{s}_{1} \cdot \bar{s}_{1}$ such that $\bar{s}_{1} \in S_{T_{d}}^{\mathcal{S}_{d}}$ with $\ln \left(\bar{s}_{1}\right)=3(d-1)$. In this case the element $\bar{s}_{1}$ is uniquely determined by its product $\alpha\left(\bar{s}_{1}\right)=\alpha\left(\widetilde{s}_{1}\right)^{-1} \alpha(s)$.

In the second part of the proof of Theorem 3.2 in [1] it was proved that for a such factorization $s=\widetilde{s}_{1} \cdot \bar{s}_{1}$ there is another factorization $s=\widetilde{s}_{2} \cdot \bar{s}_{2}$, where again $\bar{s}_{2} \in S_{T_{d}}^{\mathcal{S}_{d}}$ has the length $\ln \left(\bar{s}_{2}\right)=3(d-1)$ and $\widetilde{s}_{2}$ is uniquely determined by the type $\tau\left(\widetilde{s}_{1}\right)$. The proof of the last statement used only properties of the semigroup $S_{T_{d}}$ and relations (11) in the factorization semigroups. Therefore, by Claims 5 and 6 , the end of the proof of Theorem 1 coincides with the second part of the proof of Theorem 2.3 in [1. Only, we must do the following changes: the elements $x_{(i, j)}$ are changed by $z_{(i, j)}$, the lengths of elements are changed by $T$-lengths, the element $h_{d, g}$ is changed by $\bar{\alpha}^{-1}\left(h_{d, g}\right)$, the semigroup $S_{T_{d}}^{\mathcal{S}_{d}}$ is changed by $S_{T_{C, d}}^{\mathcal{S}_{d}, T}$, and the homomorphism $r$ is changed by $\bar{\alpha}^{-1} \circ r$. 
However, according the request of the referee, this proof is given once again. For this purpose, denote by $h_{C, d, g}=\bar{\alpha}^{-1}\left(h_{d, g}\right)$ the image of the Hurwitz element $h_{d, g}=$ $x_{(1,2)}^{2 g} \cdot x_{(1,2)}^{2} \cdot \ldots \cdot x_{(d-1, d)}^{2}$.

Lemma 1. For any disjoint union $\left\{i_{1,1}, \ldots, i_{k_{1}, 1}\right\} \sqcup \cdots \sqcup\left\{i_{1, n}, \ldots, i_{k_{n}, n}\right\}$ of ordered subsets of $I_{d}$ the Hurwitz element $h_{C, d, 0}$ can be represented as a product

$$
h_{C, d, 0}=\left(z_{\left(i_{1,1}, i_{2,1}\right)} \cdot \ldots \cdot z_{\left(i_{k_{1}-1,1}, i_{k_{1}, 1}\right)}\right) \cdot \ldots \cdot\left(z_{\left(i_{1, n}, i_{2, n}\right)} \cdot \ldots \cdot z_{\left(i_{k_{n}-1, n}, i_{k_{n}, n}\right)}\right) \cdot \bar{h},
$$

where $\bar{h}$ is an element of $S_{T_{C, d}}^{\mathcal{S}_{d}, T}$.

Proof. It directly follows from Lemma 2.9 in [1] and Claim 5.

By Claim 6, the element $s$ can be represented as a product: $s=\widetilde{s} \cdot \bar{s}$, where $\bar{s}$ is an element of $S_{T_{C, d}}^{\mathcal{S}_{d}, T}$ of $T$-length $k \geqslant 3\left(d-1\right.$ ) (in our case $\bar{s}=h_{C}$ and $k=3(d-1)$ ), and let $\widetilde{s}^{\prime}=x_{\sigma_{1}^{\prime}} \cdot \ldots \cdot x_{\sigma_{m}^{\prime}}$. By Proposition 2.4 in [1] and Claim 5, we have $\bar{s}=h_{C, d, 0} \cdot \bar{s}^{\prime}$.

To complete the proof of Theorem 1, let us use induction on $m$. If $m=0$ (that is, if $s \in S_{T_{C, d}}$ ), then Theorem 1 follows from Proposition 2.4 in [1] and Claim 5 .

Let $m=1$. For the canonical representative $\sigma_{m, 0}$ of type $t\left(\sigma_{m}\right)$ (the definition of the canonical representative is given in [1]), there is an element $\bar{\sigma}_{m} \in \mathcal{S}_{d}$ such that $\sigma_{m, 0}=\bar{\sigma}_{m}^{-1} \sigma_{m}^{\prime} \bar{\sigma}_{m}$. The permutation $\bar{\sigma}_{m}$ can be factorized into the product of cyclic permutations and each cyclic permutation can be factorized into the product of transpositions:

$$
\bar{\sigma}_{m}=\left(\left(i_{1,1}, i_{2,1}\right) \ldots\left(i_{k_{1}-1,1}, i_{k_{1}, 1}\right)\right) \ldots\left(\left(i_{1, n}, i_{2, n}\right) \ldots\left(i_{k_{n}-1, n}, i_{k_{n}, n}\right)\right) .
$$

Consider the element

$$
\bar{r}\left(x_{\bar{\sigma}_{m}}\right)=\left(z_{\left(i_{1,1}, i_{2,1}\right)} \cdot \ldots \cdot z_{\left(i_{k_{1}-1,1}, i_{k_{1}, 1}\right)}\right) \cdot \ldots \cdot\left(z_{\left(i_{1, n}, i_{2, n}\right)} \cdot \ldots \cdot z_{\left(i_{k_{n}-1, n}, i_{k_{n}, n}\right)}\right) \in S_{T_{C, d}} .
$$

By Lemma 1, we have

$$
h_{C, d, 0}=\bar{r}\left(x_{\bar{\sigma}_{m}}\right) \cdot \bar{h}_{m},
$$

where $\bar{h}_{m}$ is an element of $S_{T_{C, d}}^{\mathcal{S}_{d}, T}$. We have

$$
\begin{aligned}
s= & x_{\sigma_{m}^{\prime}} \cdot h_{d, 0} \cdot \bar{s}^{\prime}=x_{\sigma_{m}^{\prime}} \cdot \bar{r}\left(x_{\bar{\sigma}_{m}}\right) \cdot \bar{h}_{m} \cdot \bar{s}^{\prime}= \\
& \bar{r}\left(x_{\bar{\sigma}_{m}}\right) \cdot x_{\sigma_{m, 0}} \cdot \bar{h}_{m} \cdot \bar{s}^{\prime}=x_{\sigma_{m, 0}} \cdot \bar{r}\left(x_{\bar{\sigma}_{m}^{\prime}}\right) \cdot \bar{h}_{m} \cdot \bar{s}^{\prime},
\end{aligned}
$$

where $x_{\bar{\sigma}_{m}^{\prime}}=\lambda\left(\sigma_{m, 0}\right)\left(x_{\bar{\sigma}_{m}}\right)$. We have $\bar{s}_{1}^{\prime}=\bar{r}\left(x_{\bar{\sigma}_{m}^{\prime}}\right) \cdot \bar{h}_{m} \cdot \bar{s}^{\prime} \in S_{T_{C, d}}^{\mathcal{S}_{d}, T}$ and $\alpha\left(\bar{s}_{1}^{\prime}\right)=\sigma_{m, 0}^{-1} \alpha(s)$. Theorem 2.4 in [1] and Claim [5 imply that $\bar{s}_{1}^{\prime}=\bar{r}\left(x_{\sigma}\right) \cdot h_{C, d, g}$, where $\sigma=\alpha\left(\bar{s}_{1}^{\prime}\right)=$ $\sigma_{m, 0}^{-1} \alpha(s)$ and $g=\frac{k-l n_{t}\left(x_{\sigma}\right)}{2}-d+1$.

Now assume that Theorem 1 is proved for all $m<m_{0}$ and consider an element

$$
s=x_{\sigma_{1}} \cdot \ldots \cdot x_{\sigma_{m_{0}}} \cdot \bar{s}_{1},
$$

where the element $\bar{s}_{1} \in S_{T_{C, d}}^{\mathcal{S}_{d}, T}$ has $T$-length equal $k \geqslant 3(d-1)$. We have

$$
\begin{aligned}
& s=x_{\sigma_{1}} \cdot \ldots \cdot x_{\sigma_{m_{0}}} \cdot \bar{s}_{1}=x_{\sigma_{2}^{\prime}} \cdot \ldots \cdot x_{\sigma_{m_{0}}^{\prime}} \cdot x_{\sigma_{1}} \cdot \bar{s}_{1}= \\
& x_{\sigma_{2}^{\prime}} \cdot \ldots \cdot x_{\sigma_{m_{0}}^{\prime}} \cdot x_{\sigma_{1,0}} \cdot \bar{s}_{1}^{\prime}=x_{\sigma_{1,0}} \cdot x_{\sigma_{2}^{\prime \prime}} \cdot \ldots \cdot x_{\sigma_{m_{0}}^{\prime \prime}} \cdot \bar{s}_{1}^{\prime},
\end{aligned}
$$


where $\sigma_{j}^{\prime}=\sigma_{1} \sigma_{j} \sigma_{1}^{-1}$ and $\sigma_{j}^{\prime \prime}=\sigma_{1,0}^{-1} \sigma_{j}^{\prime} \sigma_{1,0}$ for $j=2, \ldots, m$, and the element $\bar{s}_{1}^{\prime} \in S_{T_{C, d}}^{\mathcal{S}_{d}, T}$ has $T$-length $\ln _{T}\left(\bar{s}_{1}^{\prime}\right)=k$. It follows from inductive assumption that

$$
s=x_{\sigma_{1,0}} \cdot\left(x_{\sigma_{2}^{\prime \prime}} \cdot \ldots \cdot x_{\sigma_{m_{0}}^{\prime \prime}} \cdot \bar{s}_{1}^{\prime}\right)=x_{\sigma_{1,0}} \cdot\left(x_{\sigma_{2,0}} \cdot \ldots \cdot x_{\sigma_{m_{0}, 0}} \cdot \bar{s}_{1}^{\prime \prime}\right),
$$

where the element $\bar{s}_{1}^{\prime \prime} \in S_{T_{C, d}}^{\mathcal{S}_{d}, T}$ has $T$-length $\ln _{T}\left(\bar{s}_{1}^{\prime \prime}\right)=k$. By Proposition 2.4 in [1] and Claim 5, we have $\bar{s}_{1}^{\prime \prime}=\bar{r}\left(x_{\sigma}\right) \cdot h_{C, d, g}$, where $\sigma=\alpha\left(\bar{s}_{1}^{\prime \prime}\right)=\left(\sigma_{1,0} \ldots \sigma_{m, 0}\right)^{-1} \alpha(s)$ and $g=\frac{k-l n_{t}\left(x_{\sigma}\right)}{2}-d+1$.

\section{REFERENCES}

[1] Vik.S. Kulikov: Factorization semigroups and irreducible components of Hurwitz space. Izv. Math., 75:4 (2011), 711748

[2] B. Wajnryb: Orbits of Hurwitz action for coverings of a sphere with two special fibres. Indag. Math. (N.S.), vol. 7 (1996), no. 4, $549-558$.

Steklov Mathematical Institute

E-mail address: kulikov@mi.ras.ru 